\title{
Ciclo de Mejora para la Innovación Docente aplicado en materias de un Área Técnica Arquitectónica
}

CARLOS Rivera GómEZ

Universidad de Sevilla. E.T.S. de Arquitectura. Departamento de Construcciones Arquitectónicas I crivera@us.es

ORCID: https://orcid.org/0000-0001-5324-068X D.0.1.: http://dx.doi.org/10.12795/JDU.2018.i01.66 Pp.: $1170-1190$

\section{Resumen}

La presente comunicación consiste en el relato completo de la experiencia docente del diseño y los resultados de la aplicación de un Ciclo de Mejora (CDM) en distintos temas de una asignatura del Área Técnica Arquitectónica. La viabilidad de la metodología empleada y la secuencia específica de actividades son analizadas con objeto de obtener las conclusiones precisas para lograr la mayor efectividad del aprendizaje de este tipo de materias en las que prevalecen los contenidos procedimentales y el estudio de casos y problemas. El CDM ha sido monitorizado en su integridad como un estudio específico de casos dentro del Proyecto de Excelencia del Plan Estatal 20013-16 titulado La Formación Docente del Profesorado Universitario. Progresos y Obstáculos de los Participantes en un Programa Basado en Ciclos de Mejora de su Práctica. 
Palabras Clave: Innovación docente, Construcción I, Grado en Fundamentos de Arquitectura, Docencia universitaria de áreas técnicas, Experimentación docente universitaria.

\section{Contexto}

La asignatura en la que se ha aplicado el CDM es Construcción Uno del Grado en Fundamentos de Arquitectura. Es una asignatura troncal y está situada en el primer cuatrimestre del primer año de carrera. Los contenidos de la asignatura están destinados a introducir al alumno en los rudimentos de la tecnología constructiva y su representación gráfica, así como en los aspectos relativos al acondicionamiento y las instalaciones de los edificios. El perfil de los alumnos es desigual, proviniendo de bachillerato en su mayoría, aunque también de ciclos formativos en edificación. Estos últimos abordan la asignatura con un dominio considerable de los recursos de representación gráfica constructiva. Los temas tratados durante el CDM son, por una parte, La Calidad del Aire interior y su control. Los objetivos de este tema son la intelección de los requisitos básicos de la ventilación de edificios y la lógica constructiva, de cálculo y de diseño aplicada a estos sistemas dentro del marco de la normativa. Y, por otra, una parte del tema Estructuras Horizontales Forjados Unidireccionales de Hormigón Armado, en concreto la referente al replanteo inicial de dichos forjados. Los objetivos generales de dicho tema son el conocimiento de los requisitos, funcionalidad, proceso constructivo y descripción gráfica de los elementos estructurales que integran un forjado unidireccional de hormigón armado. Se ha seleccionado dicho tema por la implicación constructiva que los sistemas de ventilación suponen en las soluciones de forjado.

Considerando, para ambos temas, la necesidad del conocimiento de la normativa a través de los documentos básicos correspondientes del Código Técnico de la Edificación [1]. 


\section{Principios Didácticos}

Los Principios Didácticos fundamentales que han primado en la concepción y desarrollo del CDM han estado inspirados por las directrices enunciadas por Blain [2] y Finkel [3] y han sido de diversa índole. En primer lugar un Propósito General de Mejora respecto al CDM impartido el curso anterior, evitando errores tácticos, de administración de tiempos y contenidos. Por otra parte también se ha tenido en consideración los relativos a la Exposición y Sistematización de Contenidos mediante el Mapa de Contenidos y Problemas del tema tratado. Asimismo se ha tenido en cuenta el principio de Vinculación de la Teoría con la Práctica. Bien sea presentando a los estudiantes ciertos hechos de la realidad conectados con el asunto estudiado (la investigación personal sobre sistemas de ventilación en sus casas o en la escuela de arquitectura) o bien proponiendo como ejemplo la solución de Problemas prácticos de la Vida Real. Se ha tenido presente el principio de Asequibilidad, tratando de acercar progresivamente al alumno a los aspectos más abstractos justificándolos en contenidos más directamente inteligibles y relacionados con su contexto habitual. También se han Jerarquizado los Contenidos, insistiendo en aquellos aspectos más complejos con objeto de que se fijen y consoliden, relegando a un segundo plano los menos importantes. La metodología ha hecho posible el principio de Equilibrar el Rol del Profesor y el Trabajo Independiente de los Estudiantes, permitiendo la motivación y activación de estos últimos y prestando la necesaria atención al cuidado de las condiciones externas del proceso. La figura del profesor ha tratado de actuar en este entorno garantizando el principio básico de Atención Individualizada del estudiante en la interacción con el grupo y con la clase en su conjunto. 


\section{Ciclo de Mejora}

El CDM ha consistido en cuatro sesiones de dos horas, hasta las ocho horas presenciales a las que han sumado unas seis horas de trabajo en casa realizado de forma autónoma por el estudiante. Considerado de forma global el ciclo ha abordado catorce horas de trabajo a realizar durante dos semanas. Las fechas que ha abarcado han sido los días 22, 26 y 29 de octubre y el 9 de noviembre de 2018.

El Objetivo General del ciclo ha sido el Aprendizaje de los conceptos teóricos, herramientas de cálculo y herramientas gráficas que permitan al alumno no sólo entender el sentido arquitectónico de los temas estudiados, sino solucionar mediante un lenguaje gráfico casos reales relativos a dichos temas.

Los Objetivos Específicos han sido: Ayudar al estudiante en el desarrollo de sus aptitudes creativas. Favorecer la evaluación continua y la autoevaluación del propio estudiante. Favorecer el paso hacia trabajo autónomo al iniciar al alumno en el aprendizaje independiente. Analizar y resolver cuestiones propias de la práctica profesional. Acercar a los estudiantes al tipo de problemas que se encontrarán en el futuro. Facilitar el aprendizaje de competencias complejas asociadas a la resolución de problemas, el trabajo en equipo o la toma de decisiones. Fomentar el trabajo en grupo. Motivar al estudiante para que aprenda a debatir. Desarrollar las habilidades de comunicación del alumno. Y experimentar un aprendizaje y evaluación ligados a hechos reales.

Respecto a las cuestiones asociadas a la Metodología del CDM se han considerado las siguientes: Analizar la jerarquización de contenidos y técnicas con el objeto de extraer los esenciales y favorecer su aprendizaje mediante las herramientas didácticas adecuadas. Realizar un diseño de las sesiones de trabajo en clase que incluya el 
material de aprendizaje necesario y una planificación del tiempo acorde con las respectivas actividades desarrolladas. Proporcionar al alumno las herramientas adecuadas para la resolución de las actividades planteadas para realizarse autónomamente fuera del aula. Posibilitar los medios que permitan una inmediata y adecuada valoración del aprendizaje del estudiante a lo largo del CDM. Registrar el transcurso de las sesiones para poder obtener la información pertinente que permita analizar el CDM en su conjunto como parte de una experiencia de mejora docente.

A continuación se muestra el diseño completo del Segundo Ciclo de Mejora. En primer lugar los Mapas de contenidos y Problemas (MCyP) (Figuras 1 y 2). En el primer mapa se detalla la relación de contenidos: conceptos relativos a la necesidad de renovar el aire interior; conocimiento de los distintos sistemas de renovación del aire; conceptos relacionados con las normativas de aplicación y conceptos relacionados con los elementos del diseño de los sistemas de ventilación y conocimiento de las herramientas procedimentales relativas al cálculo y dimensionado de los elementos del sistema. En el segundo mapa se trata el tema de las estructuras horizontales centrado en la tipología de forjados unidireccionales de hormigón armado (HA), comprensión de elementos y trazado gráfico.

Jornadas de Formación e Innovación Docente del Profesorado | № 1 (2018) Esta obra se distribuye con la licencia Creative Commons 


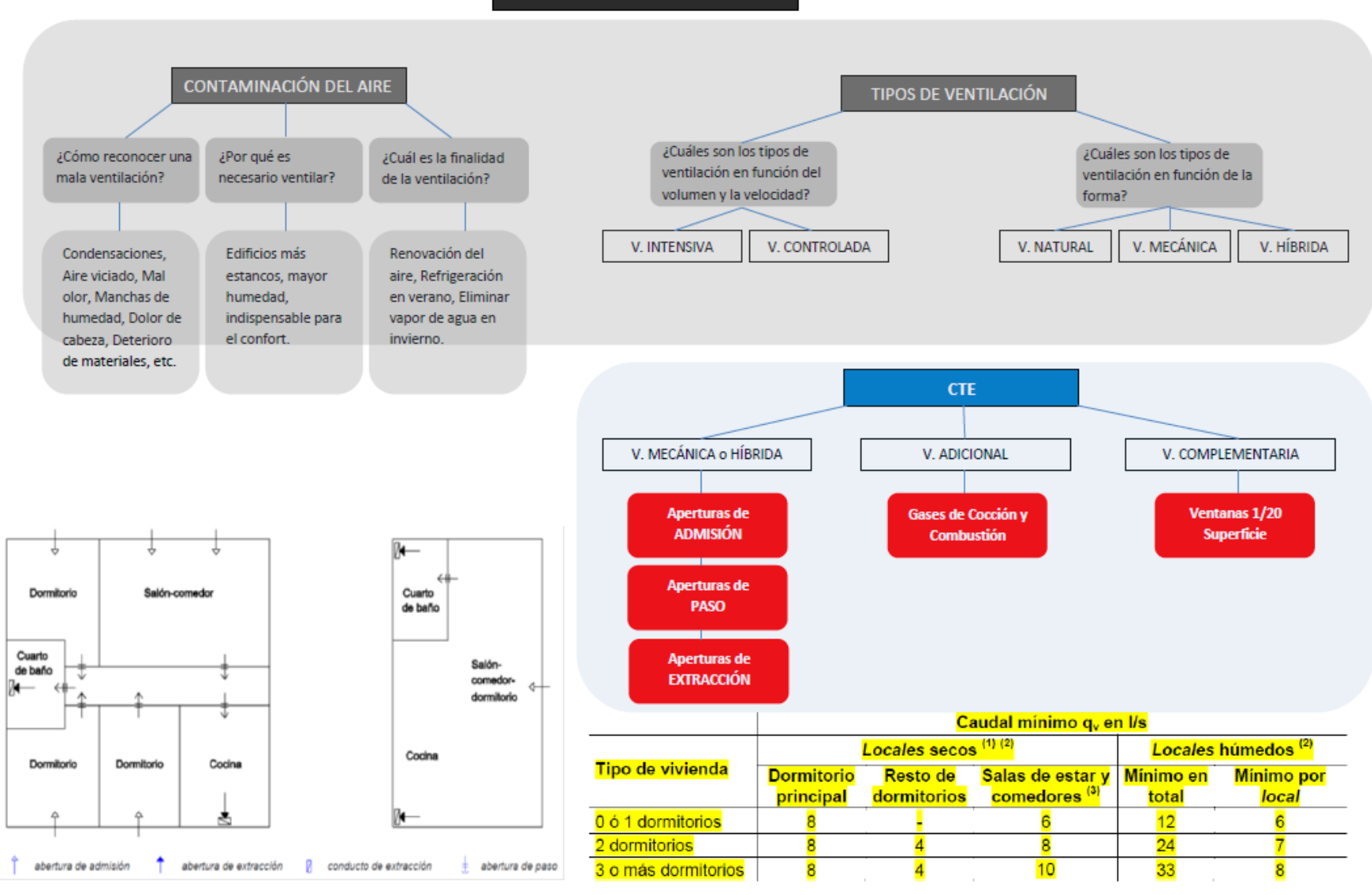

Figura 1. Mapa de Contenidos y Problemas del Tema: Calidad del Aire Interior

Jornadas de Formación e Innovación Docente del Profesorado | № 1 (2018)

(c) (i) $\ominus$ Esta obra se distribuye con la licencia Creative Commons (c) ${ }_{\mathrm{BY}} \mathrm{NC} \mathrm{ND}$ 


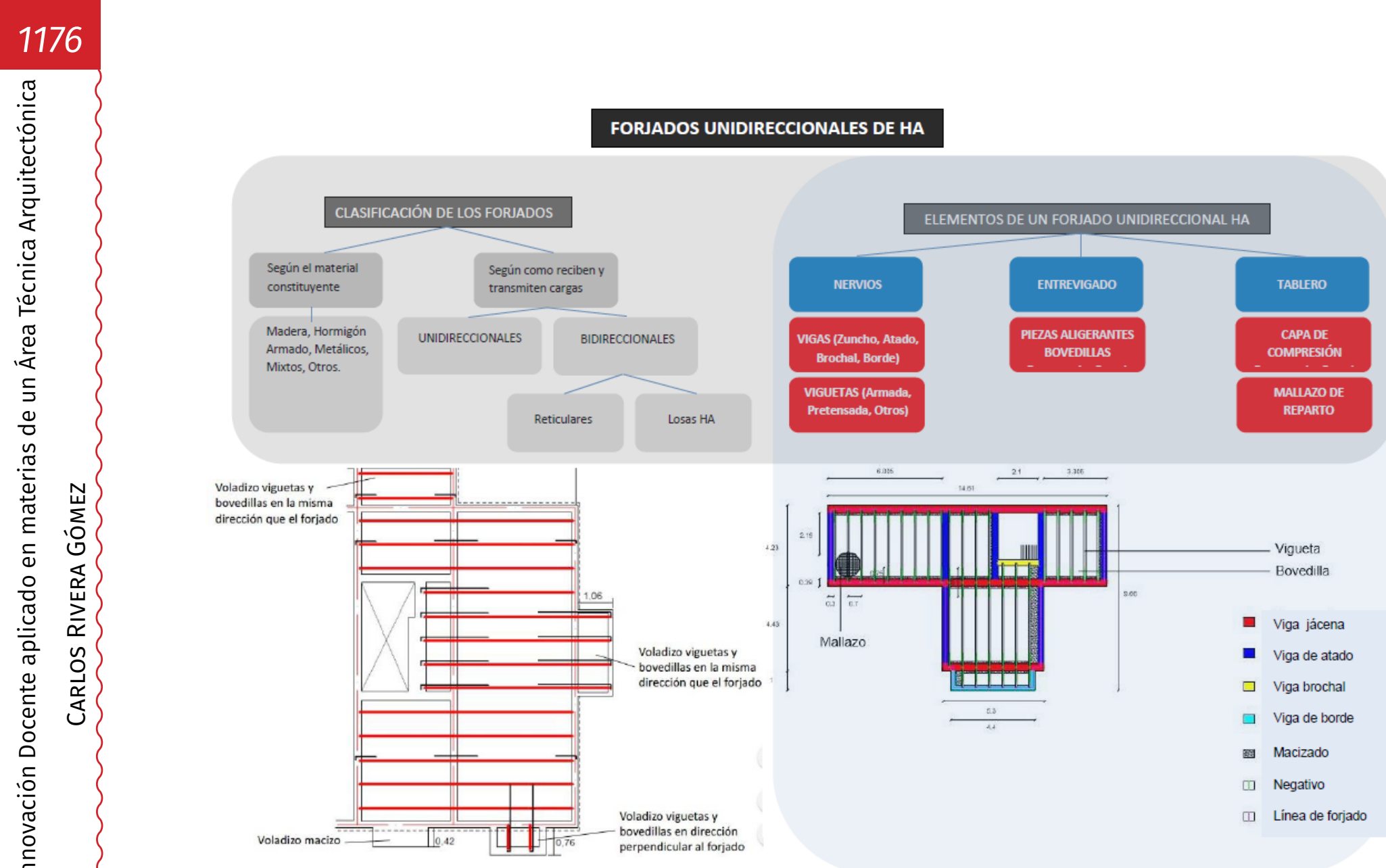

Figura 2. Mapa de Contenidos y Problemas del Tema: Forjados Unidireccionales de HA

Jornadas de Formación e Innovación Docente del Profesorado | № 1 (2018)

(c) $(1)$ Esta obra se distribuye con la licencia Creative Commons

cc) (†) $\Theta$ Reconocimiento-NoComercial-SinObraDerivada

Internacional (CC BY-NC-ND 4.0.) 
El Modelo Metodológico (figura 3) y la Secuencia de Actividades (tabla 1) y muestran en detalle la concatenación y organización de actividades en el ciclo.

El Modelo metodológico usado en el CDM sobre la misma asignatura en el curso 2017/18 presentó una serie de inconvenientes. El primero de ellos es que se partía en cada sesión de un planteamiento inicial excesivamente reducido y se concedía mucho tiempo al trabajo en grupo. Esta dinámica no se mostró adecuada para todas las jornadas del CDM, ya que el alumno no está acostumbrado a este tipo de actividades y posiblemente sea más práctica una introducción al trabajo autónomo más gradual. Por ello se propuso en el presente CDM dividir las cuatro sesiones en dos bloques. Cada uno de ellos incluye una primera sesión de trabajo en la que el trabajo en grupo es más de tipo conceptual, relativo a los conceptos teóricos del ciclo, utilizando la herramienta de Aprendizaje Basado en Problemas (ABP) que requiere un menor nivel de información procedimental previo y que corresponde a las partes sombreadas en gris en el MCyP. Y una segunda fase en que se aplica ese contenido junto a una serie de herramientas gráficas para aplicarlos a procedimientos de cálculo y descripción de soluciones gráficas aplicadas a casos prácticos, utilizando para ello la herramienta de Aprendizaje Basado en Casos (ABC), y que corresponde a las partes sombreadas en azul en el MCyP (fig.3).

Jornadas de Formación e Innovación Docente del Profesorado | № 1 (2018) Esta obra se distribuye con la licencia Creative Commons 

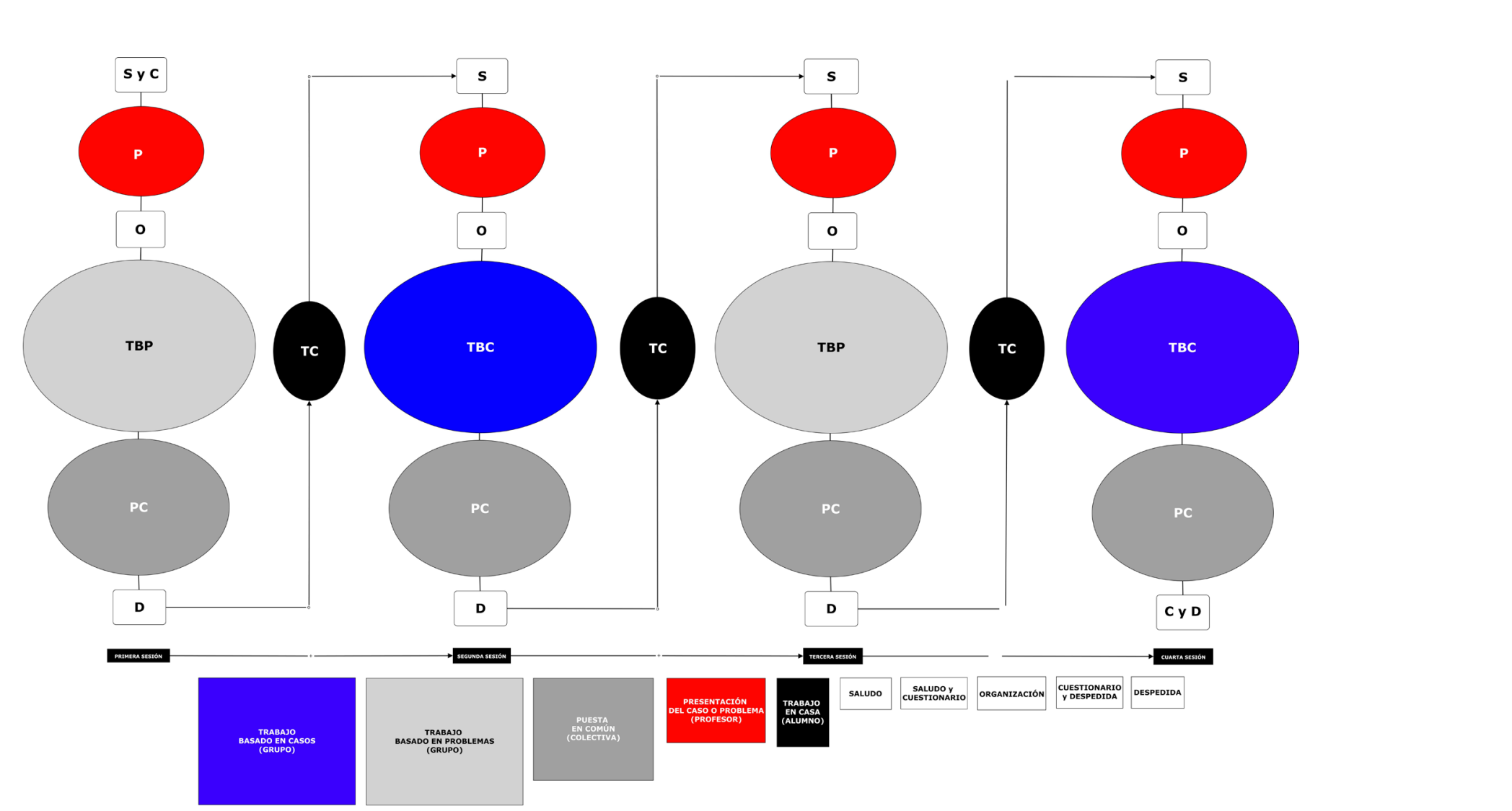

Figura 3. Modelo Metodológico y leyenda de actividades propuesta para el CDM.

Jornadas de Formación e Innovación Docente del Profesorado | № 1 (2018)

(C) Esta obra se distribuye con la licencia Creative Commons

cc) $(1) \Theta$ Reconocimiento-NoComercial-SinObraDerivada Internacional (CC BY-NC-ND 4.0.) 
Como se puede observar en la tabla siguiente en la que se detalla la Secuencia de Actividades (tabla 1), el porcentaje de trabajo autónomo de los alumnos, siguiendo las pautas de Finkel [3], representa más del 70\% del total y las puestas en común un 15\%, por lo que las actividades en la que éstos no adoptan un rol protagonista se reduce a menos de un $15 \%$.

Tabla 1. Secuencia de Actividades propuesta para el CDM.

\begin{tabular}{|c|c|c|c|}
\hline Actividad & Descripción & Tiempo & Sesión \\
\hline \multirow[t]{2}{*}{$\mathrm{S}$ y $\mathrm{C}$} & $\begin{array}{l}\text { Saludo a los alumnos, repaso de los } \\
\text { conceptos fundamentales de la clase } \\
\text { anterior, dudas sobre ese contenido, } \\
\text { descripción del contenido de la clase } \\
\text { presente, anuncios y comunicaciones } \\
\text { sobre la asignatura. Presentación del } \\
\text { tema, contextualización en la asigna- } \\
\text { tura e indice. }\end{array}$ & $10^{-}$ & 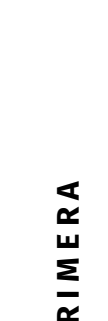 \\
\hline & Cuestionario previo & $10^{-}$ & \\
\hline$P$ & $\begin{array}{l}\text { Información sobre el ciclo de mejora. } \\
\text { Presentación del Problema a desarro- } \\
\text { llar en clase. }\end{array}$ & $5^{\prime}$ & \\
\hline $\mathrm{O}$ & $\begin{array}{l}\text { Conformación de grupos y Distribución } \\
\text { del primer Guion de Trabajo }\end{array}$ & 5 & \\
\hline TBP & $\begin{array}{l}\text { Trabajo en grupos sobre el problema } \\
\text { propuesto. }\end{array}$ & $55^{-}$ & \\
\hline$P C$ & $\begin{array}{l}\text { Presentación de los grupos, comen- } \\
\text { tarios y correcciones. Recapitulación } \\
\text { de contenidos fundamentales y du- } \\
\text { das finales. }\end{array}$ & $25^{-}$ & \\
\hline$D$ & $\begin{array}{l}\text { Descripción de la propuesta para el } \\
\text { trabajo en casa y despedida. }\end{array}$ & $10^{-}$ & \\
\hline $\mathrm{TC}$ & $\begin{array}{l}\text { Trabajo autónomo de los alumnos en } \\
\text { casa sobre la propuesta planteada. }\end{array}$ & $120^{-}$ & \\
\hline
\end{tabular}

Jornadas de Formación e Innovación Docente del Profesorado | № 1 (2018) Esta obra se distribuye con la licencia Creative Commons Reconocimiento-NoComercial-SinObraDerivada Internacional (CC BY-NC-ND 4.0.) 
S Saludo a los alumnos, repaso de los conceptos fundamentales de la clase anterior, dudas sobre ese contenido, descripción del contenido de la clase presente, anuncios y comunicaciones sobre la asignatura.

P Presentación del Caso a desarrollar en clase.

O Conformación de grupos y Distribución 5 del primer Guion de Trabajo

TBC Trabajo en grupos sobre el caso $70^{-}$ propuesto.

PC Presentación de los grupos, comen25 tarios y correcciones. Recapitulación de contenidos fundamentales y dudas finales.

D Comentarios finales y despedida. $10^{-}$

TC Trabajo autónomo de los alumnos en casa sobre la propuesta planteada. $120^{\circ}$

S Saludo a los alumnos, repaso de los conceptos fundamentales de la clase anterior, dudas sobre ese contenido, descripción del contenido de la clase presente, anuncios y comunicaciones sobre la asignatura.

P Presentación del Problema a desarro- $\quad 5$ llar en clase

O Conformación de grupos y Distribución 5 del primer Guion de Trabajo

TBP Trabajo en grupos sobre el problema 70 propuesto.

PC Presentación de los grupos, comen25 tarios y correcciones. Recapitulación de contenidos fundamentales y dudas finales.

D Descripción de la propuesta para el trabajo en casa y despedida.

$s^{\prime}$

-




\begin{tabular}{|c|c|c|c|}
\hline $\mathrm{TC}$ & $\begin{array}{l}\text { Trabajo autónomo de los alumnos en } \\
\text { casa sobre la propuesta planteada. }\end{array}$ & $120^{-}$ & \\
\hline S & $\begin{array}{l}\text { Saludo a los alumnos, repaso de los } \\
\text { conceptos fundamentales de la clase } \\
\text { anterior, dudas sobre ese contenido, } \\
\text { descripción del contenido de la clase } \\
\text { presente, anuncios y comunicaciones } \\
\text { sobre la asignatura. }\end{array}$ & 5 & \multirow{6}{*}{ 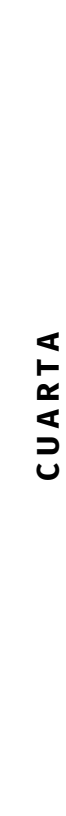 } \\
\hline$P$ & $\begin{array}{l}\text { Presentación del Caso a desarrollar en } \\
\text { clase. }\end{array}$ & 5 & \\
\hline $\mathrm{O}$ & $\begin{array}{l}\text { Conformación de grupos y Distribución } \\
\text { del primer Guion de Trabajo }\end{array}$ & 5 & \\
\hline $\mathrm{TBC}$ & $\begin{array}{l}\text { Trabajo en grupos sobre el caso } \\
\text { propuesto. }\end{array}$ & $60^{-}$ & \\
\hline PC & $\begin{array}{l}\text { Presentación de los grupos, comen- } \\
\text { tarios y correcciones. Recapitulación } \\
\text { de contenidos fundamentales y du- } \\
\text { das finales. }\end{array}$ & $25^{\prime}$ & \\
\hline Cy D & Cuestionario final y despedida. & $20^{-}$ & \\
\hline
\end{tabular}

\section{Material específico elaborado para el CDM}

Se han elaborado apuntes específicos para el CDM que sintetizan en bloques compactos de no más de cuatro páginas los temas, para ello se ha tenido en cuenta una jerarquización de contenidos sistemática (fig. 4). Los alumnos los tienen disponibles unos días antes de la clase y los utilizan para realizar los distintos ejercicios de las sesiones de trabajo siguiendo los respectivos Guiones de Trabajo en el aula. 


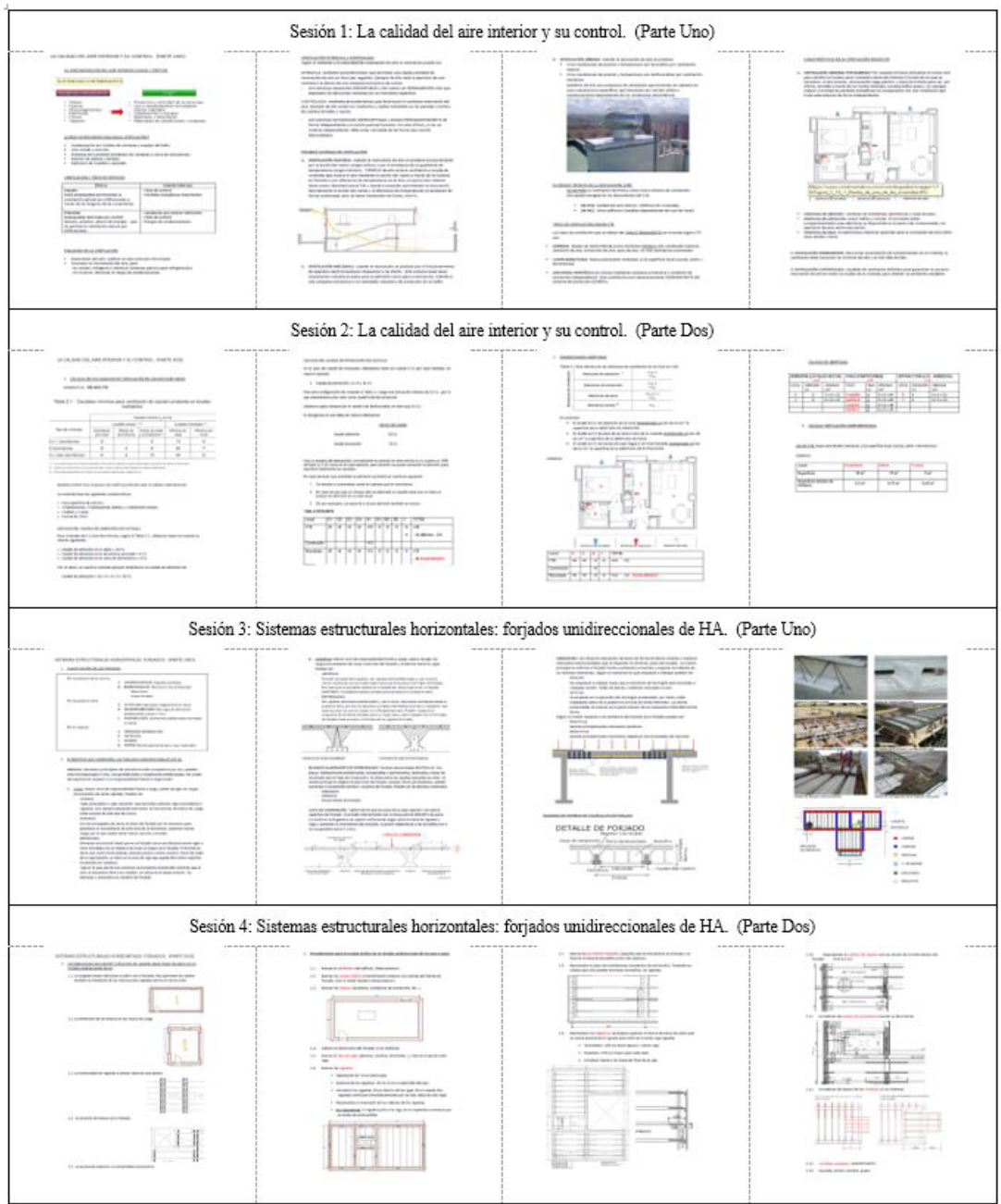

Figura 4. Apuntes suministrados a los alumnos con anterioridad a cada sesión de trabajo.

Los alumnos, trabajando en grupos, han realizado los distintos ejercicios propuestos sobre una serie de modelos comunes a lo largo de toda la realización del CDM (fig 5).

Jornadas de Formación e Innovación Docente del Profesorado I № 1 (2018)

(c) (i) $(-)$ Esta obra se distribuye con la licencia Creative Commons Reconocimiento-NoComercial-SinObraDerivada Internacional (CC BY-NC-ND 4.0.) 


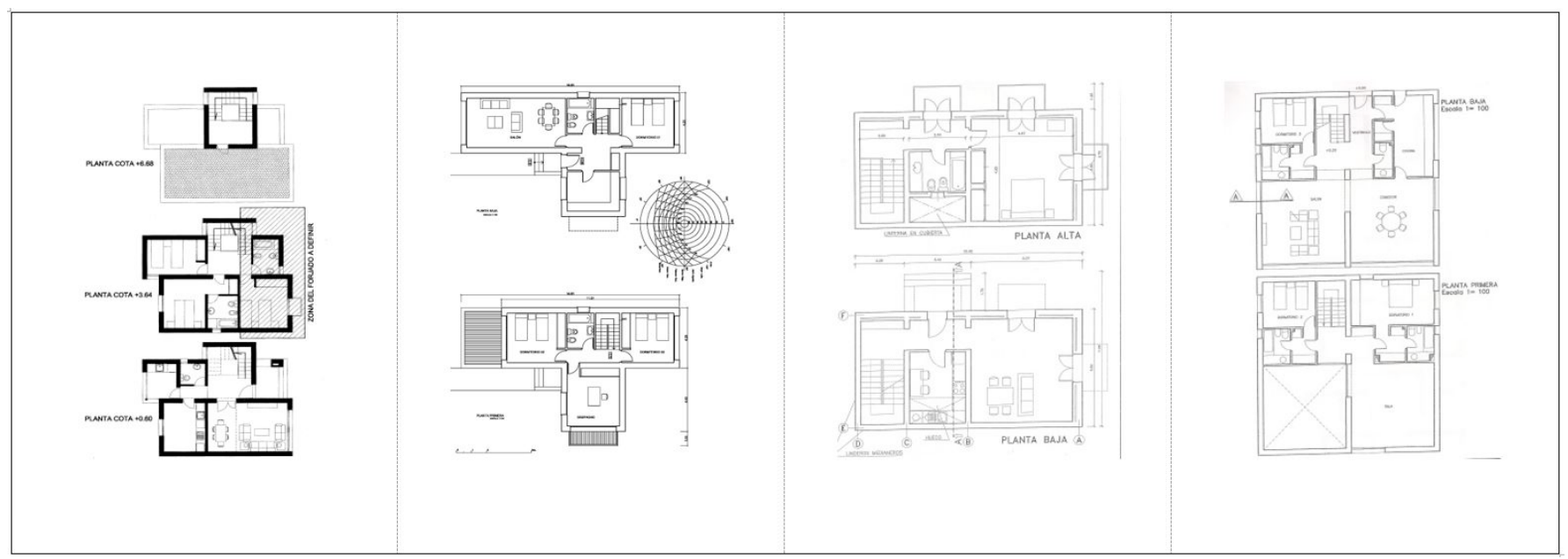

Figura 5. Ejemplos de algunos de los modelos de edificios utilizados por los alumnos a lo largo del CDM.

Para la realización del trabajo autónomo en clase se han confeccionado una serie de Guiones de Trabajo (fig. 6). 


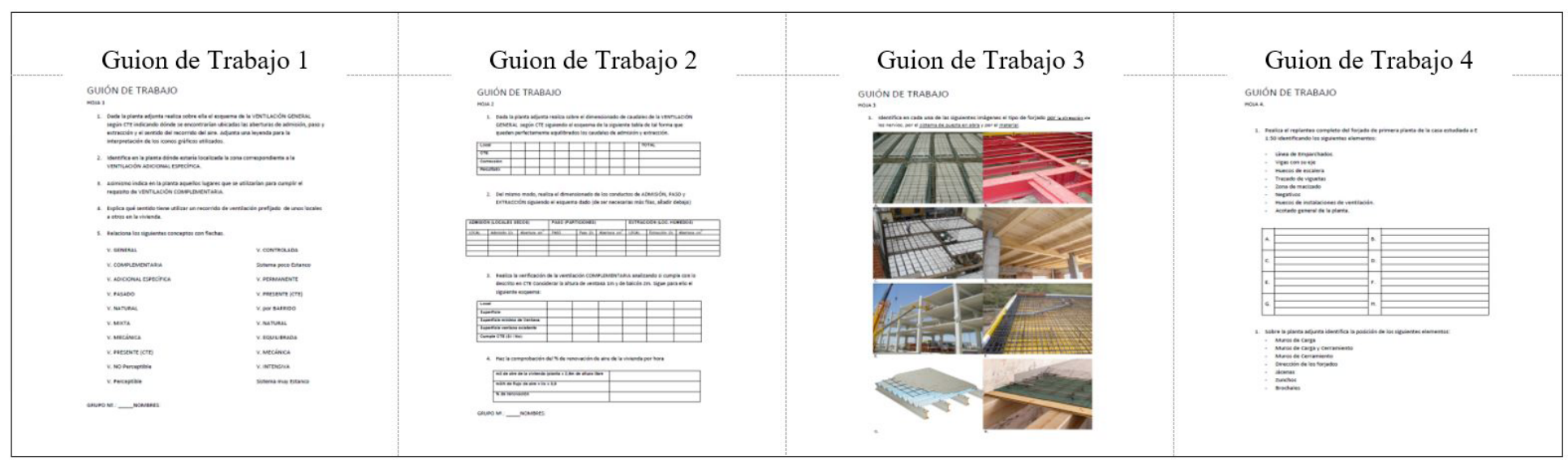

Figura 6. Guiones de trabajo utilizados por los estudiantes en cada una de las sesiones del CDM.

Asimismo, se ha elaborado un Cuestionario sobre los contenidos de los temas tratados con objeto de completar la valoración de los resultados del CDM. El cuestionario ha sido cumplimentado por los alumnos al principio y al final del CDM. En aras de la operatividad y para ser coherentes con el tiempo asignado a esta actividad en el CDM el cuestionario, a demás de representativo de los contenidos teóricos y prácticos de los temas abordados, ha sido diseñado con preguntas cortas de tal forma que se pueda cumplimentar en poco tiempo sin consumir excesivos minutos del CDM. 


\section{Relato de las Sesiones}

El CDM se ha llevado a cabo en un grupo de unos treinta alumnos, en horario de tarde y con docencia los lunes y viernes en tres sesiones de dos horas. El siguiente Diario de Sesiones es un resumen de la experiencia que contiene referencias a lo vivido en el aula. Las sesiones han sido grabadas integramente por ser tratado este CDM como un caso de estudio dentro del Proyecto de Excelencia del Plan Nacional de Investigación titulado: La Formación Docente del Profesorado Universitario. Progresos y Obstáculos de los Participantes en un Programa Basado en Ciclos de Mejora de su Práctica. Antes del comienzo del CDM, como parte del estudio, se grabó una entrevista de unos 90 minutos relativa a la concepción general y planteamientos iniciales del CDM. Están previstas, asimismo, unas encuestas a los estudiantes sobre su experiencia en el CDM y una visualización de las grabaciones de las sesiones con objeto de debatir en detalle distintos aspectos de las técnicas didácticas empleadas.

Al ser similar el desarrollo de las cuatro sesiones del CDM, con la salvedad de la inclusión del cuestionario en la primera y la última de ellas, es posible realizar una narración integrada del conjunto de las sesiones de trabajo del CDM. En síntesis las sesiones han comenzado con una breve introducción al trabajo diario por parte del profesor y una explicación del Guion de trabajo en relación a la documentación remitida a los estudiantes unos días antes del comienzo de cada una de las sesiones. A continuación se ha procedido al trabajo autónomo en grupo, fase a la que ha sucedido una puesta en común en la que ha participado toda la clase, guiada por el profesor, en la que se han subrayado los contenidos más importantes abordados en cada una de las sesiones. La culminación de la clase ha sido una indicación a la siguiente sesión en cuanto a contenidos y trabajo a realizar, anunciando a los alumnos el día en que tendrán disponibles los apuntes de trabajo 
previos a la sesión. La fluidez del transcurso de las sesiones, la concentración de los estudiantes durante la sesión, la ausencia de tiempos muertos y la participación de todos los integrantes de cada grupo han generado un clima de trabajo mantenido durante toda la duración del CDM.

\section{Evaluación del Aprendizaje de los Estudiantes}

En este apartado se muestra la evaluación comparativa del aprendizaje de los estudiantes en base a los resultados previos y posteriores al CDM. Tras la realización del Ciclo se procedió a comparar los resultados de los Cuestionarios Inicial y Final que contenían preguntas sobre cuestiones fundamentales de los temas tratados. A continuación se muestran dichos resultados en forma de Escalera de Conocimiento y Aprendizaje. La muestra considerada para el estudio fue un total de 28 encuestas individuales. Se ha unificado en una única Escalera el conjunto de las respuestas a las nueve preguntas incluidas en los Cuestionarios. En la siguiente gráfica (fig. 7) se muestran los porcentajes de los alumnos que se encuentran en cada peldaño, identificando a qué contenido se refiere cada nivel. Del análisis de estos resultados se desprende las dificultades relativas de aprendizaje para pasar de un nivel a otro superior y para obtener un grado entre adecuado y óptimo de conocimientos en relación a la materia abordada en el CDM. En color azul la escalera inicial y en rojo la final. Trazándose la línea de nivel de conocimientos adecuados se observa que, antes del CDM, este nivel estaba en un $29,5 \%$, debido fundamentalmente a la presencia de alumnos repetidores o procedentes de Ciclos Formativos en Edificación. Después del CDM este porcentaje pasa a ser del $61,5 \%$, estando otro $26,5 \%$ próximo al nivel de conocimientos satisfactorios. Lo cual implica una alta potencialidad para superar dicho nivel. 


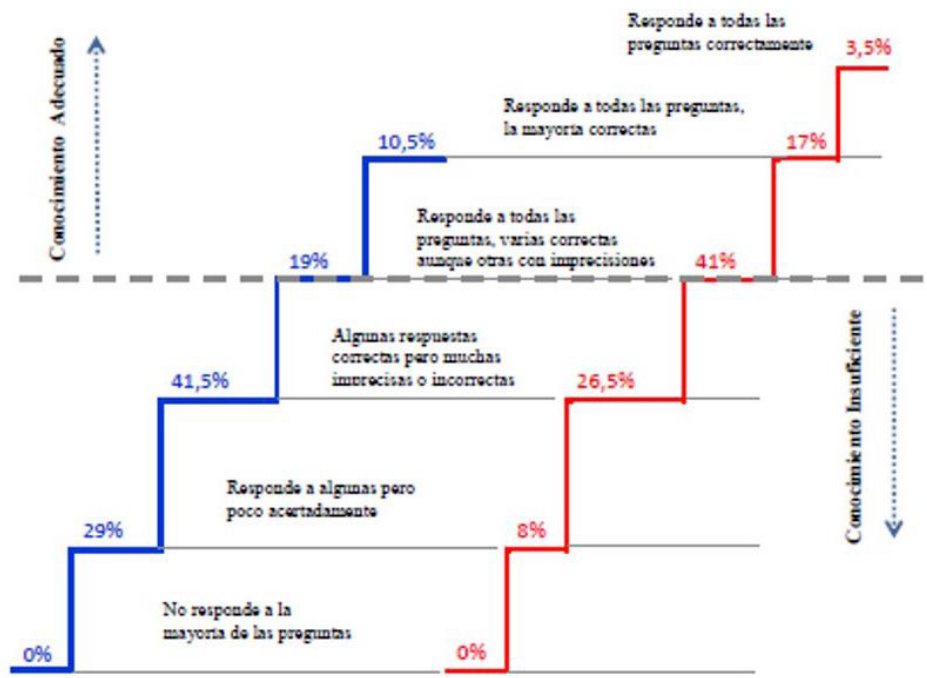

Figura 7. Escalera de Conocimiento y Aprendizaje elaborada en base a las respuestas a los Cuestionarios Inicial y Final.

\section{Evaluación del Diseño puesto en práctica}

\section{Avances Conseguidos en el Ciclo De Mejora}

El CDM ha permitido romper la dinámica habitual de la clase magistral a la que están acostumbrados los alumnos, les ha obligado a realizar un trabajo intelectual poco frecuente en clase, les ha dado la oportunidad de participar y expresar sus propias ideas, trabajar cooperativamente en grupo y a actuar proactivamente con un alto aprovechamiento del tiempo. Las clases han resultado en general más amenas e interesantes y la secuencia de las distintas actividades ha propiciado un dinamismo mayor y una notablemente más alta conexión de los estudiantes con la materia tratada en las distintas sesiones. Las relaciones profesor-estudiante y estudiantes entre sí se han

Jornadas de Formación e Innovación Docente del Profesorado I № 1 (2018)

Esta obra se distribuye con la licencia Creative Commons Reconocimiento-NoComercial-SinObraDerivada Internacional (CC BY-NC-ND 4.0.) 
reforzado en confianza tras la experiencia del CDM, especialmente en las actividades de trabajo en grupo y puesta en común que han ocupado la mayor parte del tiempo. Se ha conseguido, además, que los estudiantes lleguen al aula con una información previa y un conocimiento aproximado de los ejercicios a tratar durante esta fase. Lo cual ha eliminado la necesidad de largas introducciones para situarles en disposición de abordar los problemas y casos planteados. El nivel de concentración del grupo ha sido sorprendentemente alto durante todas las sesiones y ello se ha reflejado no sólo en los resultados el Cuestionario fina, sino en los distintos ejercicios realizados en clase. Desde la primera sesión se han mostrado proclives a probar una nueva metodología que les concediese más protagonismo dentro del aula y esta actitud se ha ido reforzando con el avance de las sesiones el CDM.

\section{Dificultades Encontradas}

La mayor dificultad ha consistido en concebir y elaborar un material específico para las distintas sesiones del CDM. En este sentido, la experiencia en los CDM realizados el curso 2017/18 ha posibilitado ajustar este nuevo material, tanto en los modelos, como en la información previa a cada clase, como en los guiones de trabajo de cada sesión. Para ello se han simplificado los Mapas de Contenidos y Problemas destacando aquellos contenidos fundamentales y adecuando el trabajo en las sesiones a las distintas zonas del mapa. También se han elaborado resúmenes específicos de los temas que incluyes aspectos esenciales de éstos y relacionados directamente con el trabajo a realizar en la sesión inmediata. Se han seleccionado diferentes modelos de edificios para cada uno de los grupos de trabajo, adecuados todos ellos a los criterios de la tipología constructiva tratada en la asignatura. A través de un mismo modelo para cada grupo a lo largo de todo el CDM, el estudiante ha podido irse familiarizando con 
él y poner en relación aspectos relativos a los dos temas tratados en el CDM. Por último, se han diseñado Guiones de Trabajo suficientemente compactos para no diversificar excesivamente las cuestiones a resolver englobando, al mismo tiempo, todas las cuestiones relevantes, tanto teóricas como procedimentales, de cada tema. En resumen, las dificultades encontradas han estado más fuera que dentro del aula, en la preparación del Ciclo, no en el transcurso del mismo.

\section{Aspectos a Mantener como Cambios Habituales}

El CDM en su conjunto ha sido de gran utilidad, las nuevas técnicas didácticas implementadas han dinamizado la clase haciendo posible un mejor aprovechamiento de la misma. Todas estas herramientas merecen ser tenidas en consideración en el futuro, mejorándose sucesivamente según el aprendizaje derivado de la experiencia de su puesta en práctica. Es un enorme salto desde el planteamiento previo de la docencia de la asignatura, de hecho, con bastante probabilidad, tanto alumnos como profesor echaremos de menos esta dinámica en el resto de temas de la asignatura fuera del CDM. Dada la efectividad demostrada en el desarrollo del CDM del material específico elaborado, el propósito que surge a partir de aquí es seguir desarrollando dicho material para el resto de temas de la asignatura de tal forma que sea un proceso relativamente sencillo la extensión del ciclo al conjunto de la misma.

\section{Conclusiones Finales}

El CDM sirve para poner en crisis el sistema habitual basado esencialmente en el modelo de clases magistrales con alguna incursión en la solución de problemas. Las ventajas derivadas de transferir la responsabilidad de la clase a los estudiantes ha resultado sorprendentemente fácil 
y de incuestionable utilidad en términos de rendimiento del tiempo de trabajo efectivo. Esta dinámica, nueva para ellos, les sorprende y activa intelectualmente, lo cual supone un aprendizaje directo e intensivo. Por otro lado permite que el estudiante establezca una conexión personal con los contenidos. Respecto al profesor, la preparación de la clase es más trabajosa y compleja, pero su desarrollo más efectivo y gratificante. La definición del planteamiento de problemas y casos en los Guiones de Trabajo en base a los contenidos determinados como estructuradores en base a un análisis concienzudo del tema para conectarlo con la práctica profesional y establecer una estructura coherente de dichos contenidos en el Mapa general que los conecta es determinante para encajar la materia en una dinámica que dé el protagonismo al estudiante. En síntesis, una experiencia docente de enorme utilidad tanto en el plano puramente de transmisión de la información como en el de incentivar la iniciativa de los estudiantes, su compromiso con la materia y sus capacidades reflexivas, de trabajo individual y en equipo.

\section{Bibliografía}

[1] Código Técnico de la Edificación. CTE. Disponible en la web: https://www.codigotecnico.org/ (Última vez consultado Octubre 2018).

[2] Blain, K. (2007). Lo que hacen los mejores profesores de Universidad. Publicaciones de la Universitat de Valéncia. Valencia, España. ISBN: 9788437066677

[3] Finkel, D. (2008). Dar clase con la boca cerrada. Publicaciones de la Universitat de Valéncia. Valencia, España. ISBN: $978-84-370-7268-5$ 